\title{
Case Report and Review of the Literature
}

\section{Pituitary Abscess: Two Case Reports and Review of the Literature}

\author{
Y. Sánchez Medina ${ }^{*}$, J. Domínguez ${ }^{1}, J_{\text {. Artazkoz }}^{3}$ and M. Boronat ${ }^{2}$ \\ ${ }^{l}$ Neurosurgery Unit, Nuestra Señora de Candelaria University Hospital, Tenerife, Spain \\ ${ }^{2}$ Endocrinology Unit, Insular de Gran Canaria University Hospital, Gran Canaria, Spain \\ ${ }^{3}$ Otorhinolaringology Unit, Insular de Gran Canaria University Hospital, Gran Canaria, Spain
}

\begin{tabular}{l} 
A R T I C L E I N F O \\
\hline Article history: \\
Received: 9 January, 2021 \\
Accepted: 26 January, 2021 \\
Published: 10 February, 2021 \\
\hline Keywords: \\
Pituitary abscess \\
hypophysitis \\
infection \\
transsphenoidal approach
\end{tabular}

\begin{abstract}
A B S T R A C T
Pituitary abscesses are infrequent entities, often forgotten and hence rarely included in the differential diagnosis of sellar lesions. However, given that they share the clinical manifestations of other more common pathologies in this region, the treatment of pituitary abscesses is usually performed correctly without demonstrating a relevant delay in therapeutic decisions when surgical options are considered as the alternative of choice. Two patients with intrasellar expansive processes and endocrinological alterations who were diagnosed intraoperatively as having pituitary abscesses are presented. In both cases, transsphenoidal surgery was performed; adjuvant antibiotic treatment was established for just one of them. Both patients are currently without symptoms due to mass-effect; one of the patients continues exhibiting residual endocrinological alteration and no signs of relapse in imaging studies.
\end{abstract}

() 2021 Yanire Sánchez Medina. Hosting by Science Repository.

\section{Introduction}

Pituitary abscesses are a pathology that have been insufficiently described in the literature. Although a presumptive diagnosis is difficult to make and there are occasions when there are important consequences due to not receiving timely treatment, early surgery, which on most occasions is destined in treating other incorrect diagnosis avoids elevated numbers of associated morbimortality.

\section{Case 1}

The patient is a 50-year-old woman with a history of primary amenorrhea lasting until age 21 who is receiving substitutive hormonal treatment with oral contraceptives. She required the administration of gonadotropins to induce pregnancy, presenting postpartum oligomenorrhea lasting until menopause at age 42. In August 2009, at age 49, she was evaluated due to 9-month clinical history of 5-10 kg weight loss, fatigue, anorexia, and nausea, as well as several syncopal episodes associated with hypotension and hypoglycemia. She had no history of infectious processes. She experienced polyuria and polydipsia. Examinations and testing were inconclusive $(153 \mathrm{~cm}, 50 \mathrm{~kg})$, revealing no evidence of known pathologies. Requested hormone studies were indicative of partial hypopituitarism (TSH 1,15; free T4 0,27; Plasma cortisol 2,74; Prolactin 9,81; Undetectable LH; FSH 0,36; Undetectable IGF-1). Magnetic resonance imaging (MRI) showed an increase in the volume of the pituitary gland, which extended itself in a tent shape through the suprasellar area until reaching the proximity of the optic chiasm, especially in the posterior region of the gland, where greater contrast enhancement is seen (Figure 1). A visual field examination ruled out optic tract affliction, and the rest of the tests requested, such as bone densitometry, chest X-ray, abdominal ultrasound, were normal.

Finally, in April 2010, it was decided that the patient would be transferred to the Department of Neurosurgery for evaluation and surgical treatment that would lead to the volumetric decrease of the lesion as well as histopathological examination that could shed light on a diagnosis. A transsphenoidal approach was used, during which, after dural opening, the discharge of purulent material was observed. Microbiological studies were negative, and the results of the histopathological examination showed mostly material with fibrinohematic characteristics. The patient received post-surgical empirical antibiotic treatment with vancomycin, clindamycin and ceftazidime for 15 days, with all cultures being negative. After following-up for two years, during which the patient required substitutive hormonal therapy as the previous endocrinological

${ }^{*}$ Correspondence to: Yanire Sánchez Medina, Department of Neurosurgery, Nuestra Señora de Candelaria University Hospital, Carretera del Rosario s/ $n$, 38010, Santa Cruz de Tenerife, Spain; E-mail: yanire.sm@hotmail.com 
symptoms persisted, and with favourable control radiological studies in previous MRI (Figure 2), an image suggestive of recurrence of the previous lesion was noticed in a new control MRI of the brain, with no

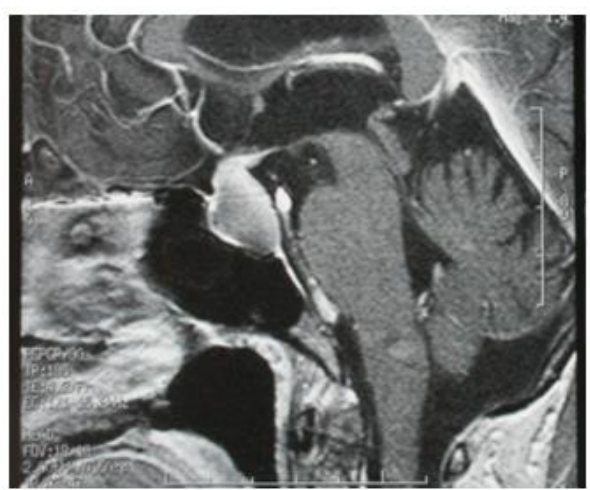

evidence of clinical modifications regarding endocrinological, ophthalmological or neurological condition (Figure 3).



Figure 1: Pre-surgical images that show intrasellar lesion with scarce contrast enhancement. Swelled pituitary stalk with no evidence of posterior pituitary lobe.
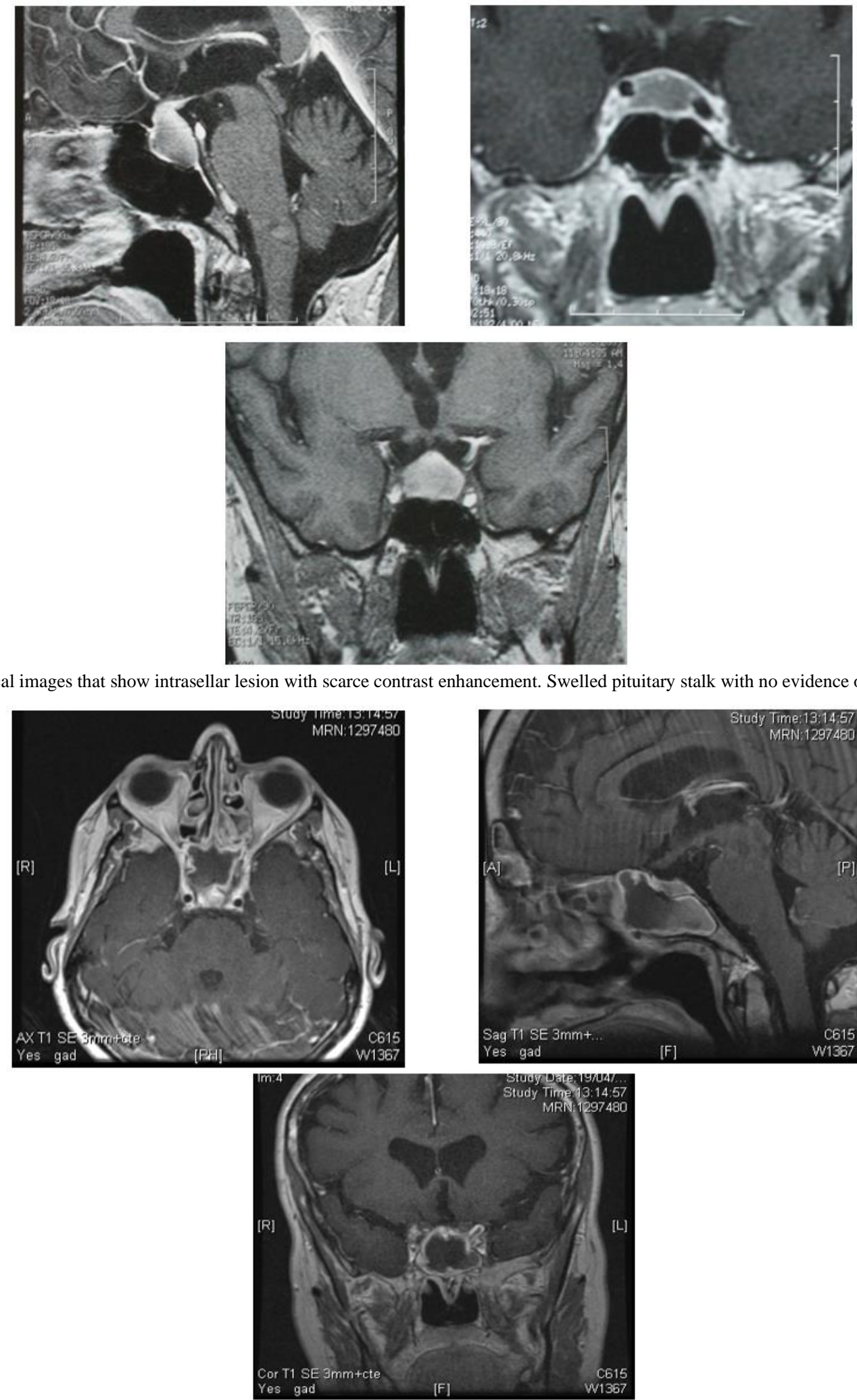

Figure 2: Post-surgical images where cystic lesion with wall enhancement is observed. Wall enhancement is associated with break in continuity with the sella turcica floor and the anterior pituitary lobe, which shows no significative enhancement with the exception of its periphery. All this is compatible with pituitary necrosis. 

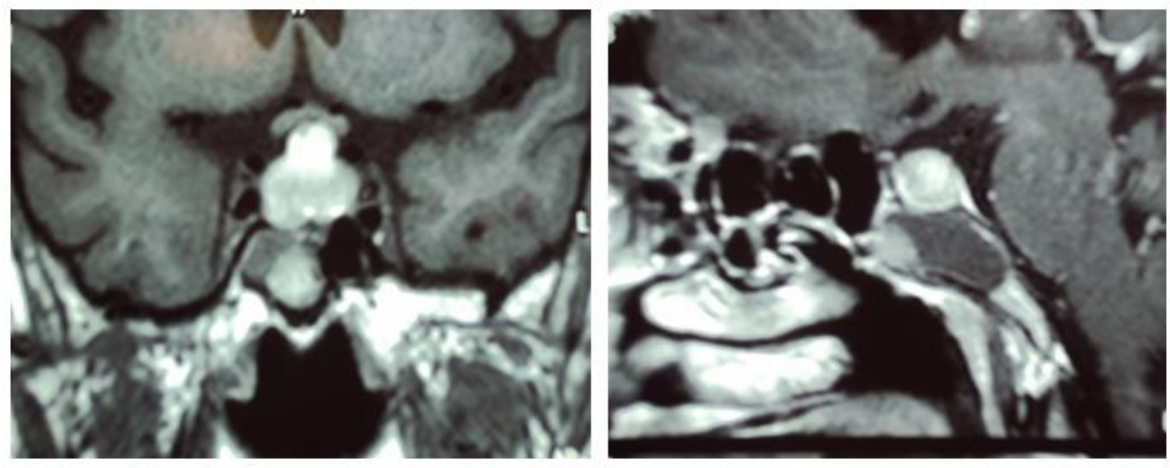

Figure 3: Recurrence images shows hyper-intense in $\mathrm{T} 2$ with intensive enhancement after the administration of intravenous contrast and suprasellar component.

A new surgical intervention was performed via transnasal endoscopic approach in which the sphenoidal sinus was found to contain mucopurulent material. After the aspiration of the content, the sellar floor was explored and found intact, without evident communication with the sinus and with partial ossification (fragments of the vomer used in the reconstruction of the floor in earlier surgery). After dural incision, discharge of creamy, very thick (pasty consistency) purulent material of
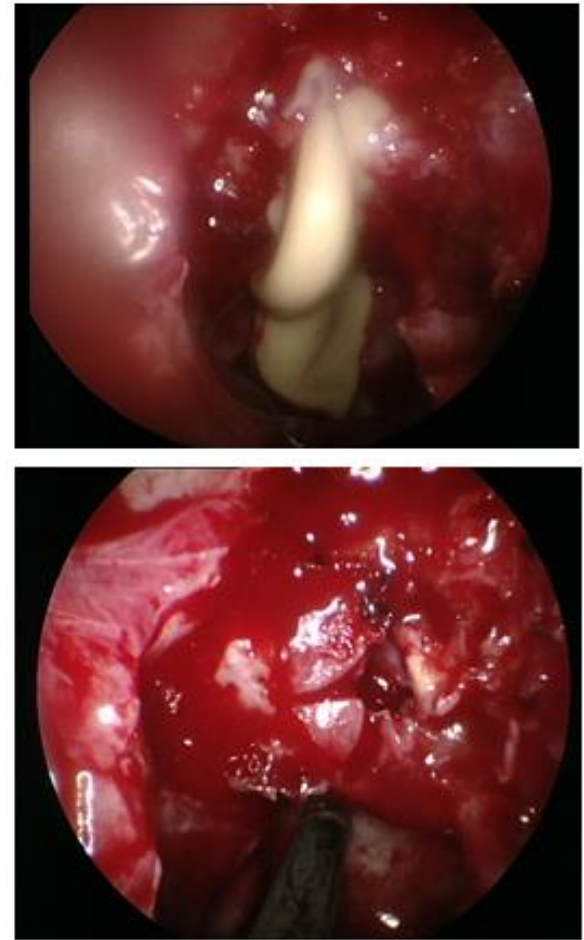

a yellowish-white colour was observed. The patient has been on empirical antibiotic therapy for 15 days and, as in earlier surgery, there is no evidence of pathogens in intraoperative culture samples (Figure 4). Actually, after 12 months of follow-up, the patient remains clinically stable, with the previously described endocrinological alteration and with favourable radiological control (Figure 5).
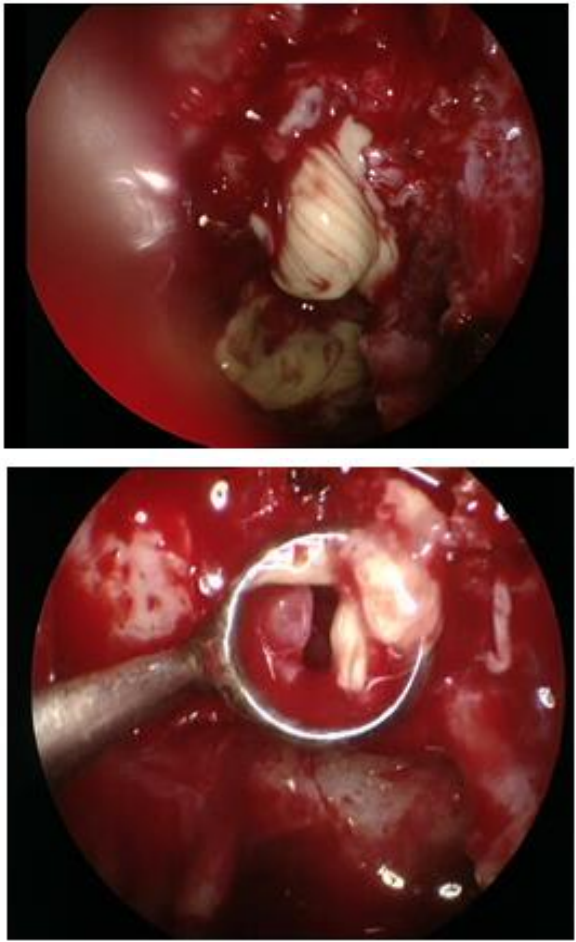

Figure 4: After dural incision, discharge of creamy, very thick (pasty consistency) purulent material of a yellowish-white colour was observed.

\section{Case 2}

A 45-year-old male patient with a year-long clinical history of impotentia coeundi and progressive weight loss with the later addition of cephalea, general fatigue and a progressive decrease in visual acuity. Physical examination showed bilateral decrease in visual acuity $(0,2 \mathrm{LE}-$ $0,1 \mathrm{RE}$ ). The analytical study showed panhypopituitarism with normal levels of prolactin (FSH 0,22; LH<0,07; Cortisol 2,21; Prolactin 12,62; IGF1 58,3; Testosterone $<0,08$; and Basal Cortisol 2,21). In the MRI of the brain, a sellar lesion with associated cystic component and significant suprasellar extension compromising the optic tract was observed (Figure 6). With a diagnosis of pituitary macroadenoma with signs of intratumoral necrosis, early surgery was chosen, and a transsphenoidal approach was performed. Moderate amounts of purulent material was discharged when moderate pressure was applied. The sample sent for microbiological testing did not evidenced any pathogen and the histopathological study showed fragments of abscess wall, with signs of chronicity, necrotic and hemorrhagic tissue. 


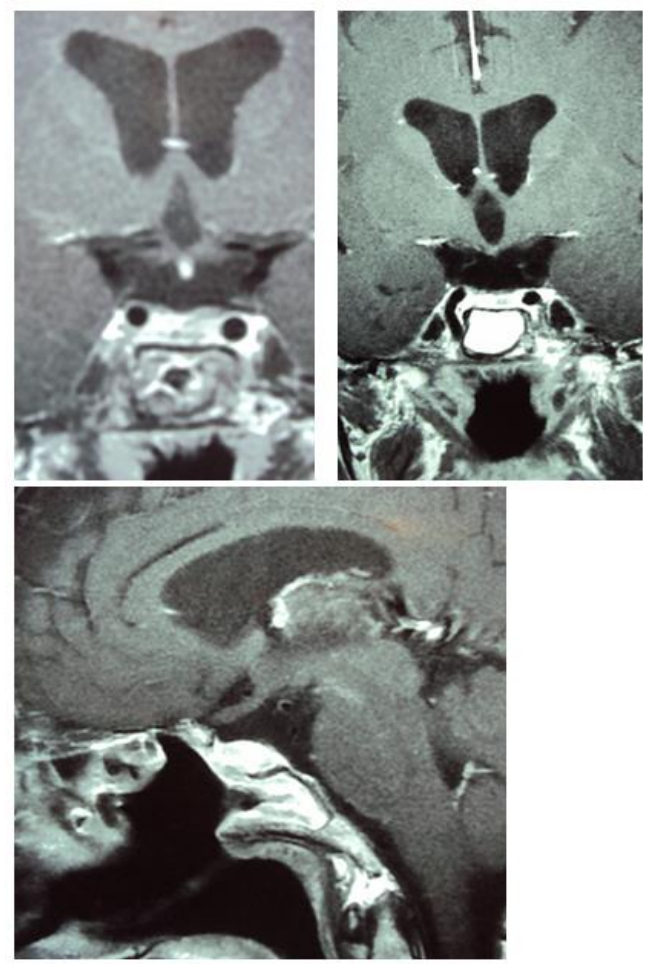

Figure 5: Images 10 months after surgery where cystic lesion is observed without enhancement compatible with fibrosis. It also shows lost of suprasellar component.

In this case, no posterior antibiotic treatment was established, opting instead only for regular postoperative prophylaxis in pituitary surgery with two doses of clindamycin $300 \mathrm{mg} / 12 \mathrm{~h}$ and four doses of cefuroxime $750 \mathrm{mg} / 6 \mathrm{~h}$. After following up for 12 months, the patient completely
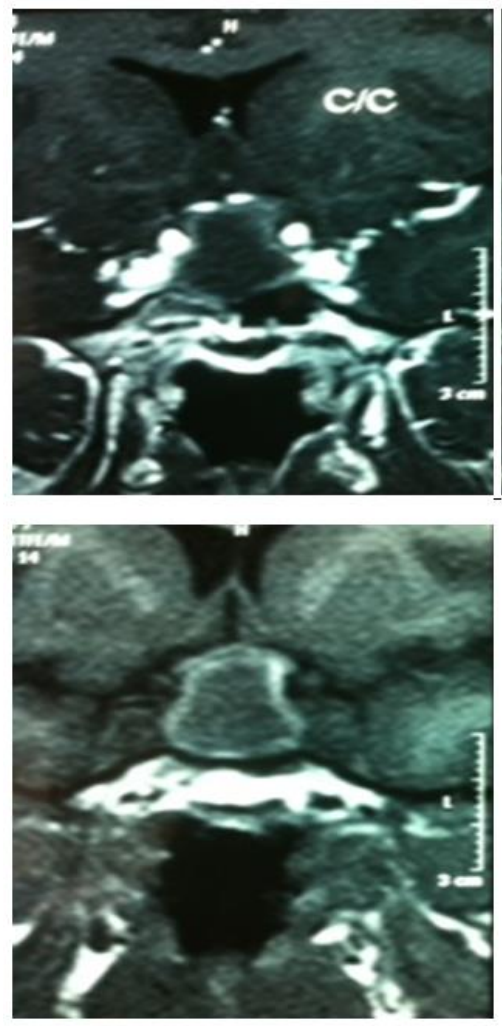

recovered from his visual deficit, with a return to normal visual acuity and field of vision, although he continues substitutive hormonal treatment due to the persistence of his panhypopituitarism, with favourable radiological control (Figure 7).

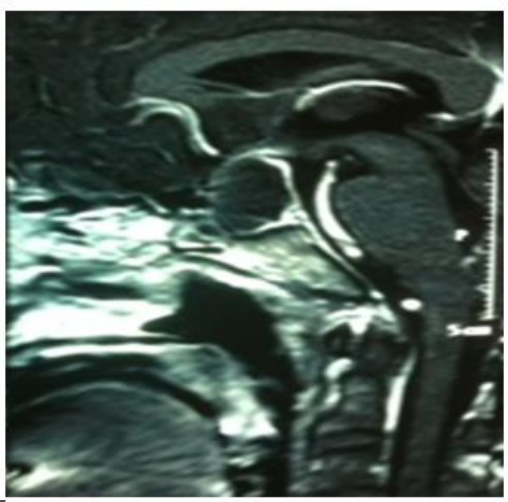

Figure 6: Pre-surgical brain MRI showing sellar lesion with associated cystic component and ample suprasellar extension with compromise of optic tract. 

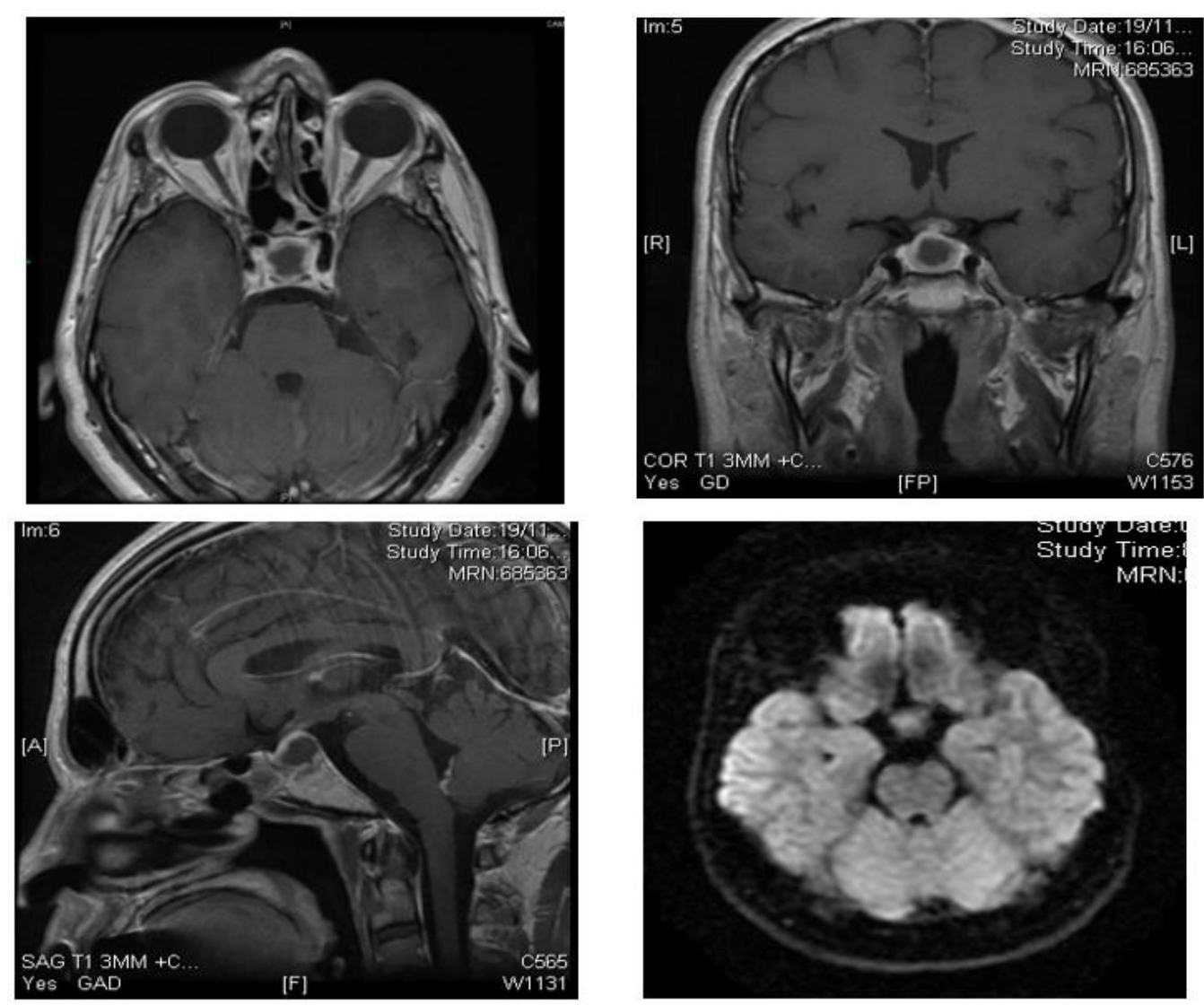

Figure 7: Brain MRI six months later showing residual intrasellar cystic lesion with wall reinforcement.

\section{Discussion}

Pituitary abscesses are an infrequent disease that constitutes less than $1 \%$ of total pituitary pathologies [1]. In 1914, Simmonds was the first to describe a case of infectious lesion in the sellar region and since few are the cases that have been registered in the literature, with Vates et al. presenting the most with a total of 24 published cases in 2001 [1]. The mechanism responsible for this pathology could be the direct extension of a previous adjacent infection such as meningitis, sinusitis, thrombophlebitis, etc., or after the hematogenous dissemination from a distant septic focus. There are various risk factors that have been postulated as predisposing factors, among which situations that cause immunodeficiencies, prior surgeries or radiation in the region, and a preexisting pituitary lesion (Craniopharyngioma, adenoma, or, especially, Rathke's cleft cyst). However, despite everything that has been presented, the literature reviewed shows that the majority of patients that have been studied spontaneously present the episode without prior history of infectious pathologies or known associated risk factors [2].

With regards to the presenting symptoms, pituitary abscesses present clinical manifestations similar to those seen in other afflictions localized in the sellar region. They often present a chronic, indolent course more suggestive of a neoplastic lesion than of the typical intense symptoms of acute onset seen in infectious and inflammatory processes [3]. This is the reason for the extreme difficulty of the initial diagnosis and the importance of performing correct anamnesis that helps to better target the pathology, as well as having a high degree of suspicion that includes an abscess among the different diagnostic options. Non-specific cephaleas are the most common symptom, along with alterations in vision. The literature reviewed describes that approximately $30-50 \%$ of patients present anterior pituitary hormonal deficiencies, with GH deficit the most common followed in order of frequency by the decrease in plasm level of FSH/LH, TSH and ACTH. Also, there is proof that a high number of patients presenting symptoms of Diabetes insipidus, as seen in our first case report, a finding that Sabba et al. observed in 50\% of the cases revised [4]. From an infectious point of view, in Vates' series, only $25 \%$ of patients presented signs of meningismus, and leukocytosis was seen in analytical tests in $30 \%$ of patients. All this, along with what's been described by the rest of the authors, shows that approximately $40 \%$ of cases present fever and meningismus in the initial evaluation, even though the infectious triad that typically presented itself such as fever, meningismus and leukocytosis was quite infrequent. Hence, the importance of including the option of an abscess in the differential diagnosis even when signs of infection are not present is once more stressed [2].

As for radiological diagnosis, pituitary abscesses are not generally considered in the differential diagnosis of a sellar lesion due to their low frequency and the lack of a characteristic radiological pattern. Pituitary adenomas make up $90 \%$ of sellar lesions and may appear as cysts or as images secondary to necrosis or hemorrhage [2]. The remaining $10 \%$ is constituted by an ample variety of pathologies, some with cystic components, among them abscesses with carcinomas, arachnoid and colloid cysts, craniopharyngioma, Rathke's cleft cysts, or metastasis. Distinguishing between them is, hence, not easy, as there is a known clinical likeness and a practically identical radiological presentation. 
In skull X-rays, abscesses usually present erosion or enlargement of the sella turcica of the skull. CT scans of the skull usually show a hypodense lesion with non-specific characteristics. In the Magnetic Resonance study, a hypo-intense mass in T1-weighed sequences and hyper-intense in T2, typical characteristics of cyst components, with ring enhancement after the administration of intravenous contrast. It is also known that this Magnetic Resonance sign can be modified in different circumstances due to the different content of protein, fat, or blood presented by the region studied, as well as other paramagnetic substances, making it extremely difficult to perform an accurate interpretation of imaging tests [4-7]. The pituitary stalk may appear enlarged, as in other pathologies such as Rathke's cleft cyst. However, all these characteristics are also seen in many other pituitary pathologies, such as the ones mentioned before, thus there seems to be no specific radiological sign leading towards the diagnosis of pituitary abscess. Precisely, this absence of radiological and clinical specificities explains that the majority of pituitary abscesses are diagnosed intraoperatively, with the discharge of purulent material from inside the lesion constituting the best possible diagnostic tool in these cases.

The microorganisms most commonly cultivated in the extracted material are Gram positive Cocci, although Gram-negative, anaerobic microorganism, fungi, and brucella have also been described. Despite this, it has been observed that in $50 \%$ of cases, the absence of microorganisms in the cultures obtained intraoperatively is common, with the presentation of findings characteristic of inflammatory processes in histopathological studies, as seen in the first case [8]. Likewise, there are controversies regarding whether to consider as an abscess a lesion that displays no microbial agent whatsoever. Concerning this last point, there are many authors who postulate that given the absence of any pathogen it would be incorrect to utilize the term 'abscess' to refer these cases and more accurately, to use 'cyst' or 'glandular necrosis'. However, Vates et al. understand that the sterile sample could be the result of an inadequate bacteriological technique or of having started antibiotic treatment prior to acquiring the surgical sample, and also that lesions that can be included in the differential diagnosis such as craniopharyngioma, Rathke's cleft cyst or pituitary necrosis are intraoperatively easily distinguished from an abscess by experienced surgeons. Transsphenoidal surgery is the therapeutic tool of choice as it allows for greater diagnostic accuracy, it eliminates the infectious agent responsible for the damage, and achieves decompression of the area, which helps to improve recovery from symptoms secondary to mass-effect caused by the lesion $[1,9,10]$.

It is considered advisable to begin antibiotic treatment after verifying discharge of purulent material during the surgical intervention for a period of 3 to 6 weeks, although there is no established consensus on this point. The most commonly used drugs include penicillin, thirdgeneration cephalosporins, and vancomycin, with treatment prescribed early on, although in asymptomatic patients, antibiotic treatment could be delayed until after microbiological results from samples obtained intraoperatively are acquired [11-14]. These controversies regarding antibiotic treatment and its duration is evidenced in cases that do not receive adjuvant antibiotic treatment after abscess surgery. Vates presents 10 examples in which antibiotherapy was not used and results were favourable. However, as seen in our second patient, in all the cases presented, prior cultures did not show any pathogens, suggesting that in cases where microbiological samples are negative, it will be up to the surgeon to decide whether to start antibiotic treatment or not. The prognosis for patients who do not receive any treatment is poor, with a $40 \%$ mortality rate. As for those who receive proper treatment, the effects seen will initially depend on the predominant clinical signs as surgery and antibiotic treatment have a curative effect on cephalea and visual alterations but small effects on established hormonal deficits [11, 15].

\section{Conclusion}

Few reviews have been contributed to the literature regarding pituitary abscesses. Generally, these represent entities that are diagnosed intraoperatively in the majority of cases unless there is a prior high-level suspicion. Regardless, transsphenoidal evacuation is considered the treatment of choice, leading to accurate therapeutic management generally despite poor diagnostic presumption.

\section{Conflicts of Interest}

None.

\section{REFERENCES}

1. Vates GE, Berger MS, Wilson CB (2001) Diagnosis and management of pituitary abscess: a review of twenty-four cases. J Neurosurg 95 : 233-241. [Crossref]

2. Dalan R, Shing Leow MK (2008) Pituitary abscess: our experience with a case and a review of the literature. Pituitary 11: 299-306. [Crossref]

3. Rao S, Rajkumar A, Kuruvilla S (2008) Sellar lesion: Not always adenoma. Ind J Pathol Microbiol 51: 269-270. [Crossref]

4. Sabba P, Bonardel G, Herve R, Marjou F, Hor F et al. (1999) CT and MRI findings in primitive pituitary abscess; a Case Report and review of literature. J Neuroradiol 26: 196-199. [Crossref]

5. Guigui J, Boukobza M, Tamer I, Guichard JP, Wyplosz B et al. (1998) Case Report: MRI and CT in a case of pituitary abscess. Clin Radiol 53: 777-779. [Crossref]

6. Shirakawa J, Takeshita T, Miyao M, Orimo S, Terauchi Y et al. (2009) Pituitary abscess with panhypopituitarism showing $\mathrm{T} 1$ signal hyperintensity of the marginal pituitary area: a non- invasive differential diagnosis of pituitary abscess and pituitary apoplexy. Inter Med 48: 441-446. [Crossref]

7. Wolansky LJ, Gallagher JD, Heary RF, Malantic GP, Dasmahapatra A et al. (1997) MRI of pituitary abscess: two cases and review of the literature. Neuroradiology 39 : 499-503. [Crossref]

8. Sahjpaul RL, Lee DH (1999) Infratentorial subdural empiema, Pituitary Abscess, and Septic Cavernous Sinus thrombophlebitis secondary to paranasal sinusitis: case Report. Neurosurgery 44: 864-866. [Crossref]

9. Oya S, Kumai J, Shigeno T (2009) Drainage or packing of the sella? Transsphenoidal surgery for primary pituitary abscess: report of two cases. Case Rep Med 2009: 189304. [Crossref]

10. Whalley N (1952) Abscess formation in a pituitary adenoma. J Neurol Neurosurg Psychiatry 15: 66-67. [Crossref]

11. Hanel RA, Koerbel A, Monte Serrat Prevedello D, Sydnei Moro M, Candido Araújo J (2002) Primary pituitary abscess: case report. Arq Neuropsiquiatr 60: 861-865. [Crossref] 
12. Jadhav RN, Dahiwadkar HV, Palande DA (1998) Abscess formation in invasive pituitary adenoma: case report. Neurosurgery 43: 616-619. [Crossref]

13. Uchida Y, Tsuchimochi N, Oku M, Fujihara N, Shimono N (2008) Successful treatment of pituitary abscess with oral administration of sparfloxacin. Inter Med 47: 1147-1151. [Crossref]
14. Pinaki D, Anil B, Paramjeet S, Narendra K, Ashish P et al. (2006) Pituitary abscess: report of four cases and review of literature. Pituitary 9: 267-273. [Crossref]

15. Snyder PJ (2010) Causes, presentation, and evaluation of sellar masses. 